\title{
Post laryngectomy speech rehabilitation outcome in elderly patients
}

\author{
Salvatore Cocuzza - Marco Bonfiglio $\cdot$ Calogero Grillo • \\ Luigi Maiolino $\cdot$ Mariano Malaguarnera • \\ Francesco Martines • Agostino Serra
}

Received: 2 January 2013/ Accepted: 27 February 2013

(C) Springer-Verlag Berlin Heidelberg 2013

\begin{abstract}
The aim of our work has been to evaluate the different options of tracheoesophageal voice rehabilitation in over 70-year-old patients, who had undergone laryngectomy, assessing advantages and drawbacks of this method of vocal recovery. A retrospective study has been carried out. This has included 40 subjects, all aged more than 70 years old, who have been referred to tracheoesophageal voice rehabilitation. It has been realized a phonatory fistula between trachea and esophagus with prosthesis positioning by means of a primary puncture in 18 cases and it has been realized a secondary puncture in 22 cases. The results gathered in these patients were compared with data obtained from a group made of 39 patients, less than 70 years of age that therefore represented our control group. In primary tracheoesophageal puncture (TEP), the short-term success was $67 \%$, while in the 22 cases who underwent secondary TEP, the short-term success was $64 \%$. After 2 years from TEP, the long-term success was $82.5 \%$. In the control group, the short-term success was $65 \%$ in primary TEP and $73 \%$ in secondary TEP. After 2 years from TEP, the long-term success was $77 \%$. The evaluation of the results has shown the absence
\end{abstract}

S. Cocuzza · M. Bonfiglio (凶) · C. Grillo · L. Maiolino ·

A. Serra

Department of Medical Surgical Specialties, ENT Clinic,

University of Catania, Policlinico Universitario "Gaspare

Rodolico", Via Santa Sofia, 68, 95125 Catania, Italy

e-mail: mrc.bnf@hotmail.it

M. Malaguarnera

Department of Senescence, Urological and Neurological

Sciences, University of Catania, Catania, Italy

F. Martines

Section of Otorhinolaryngology, Department of Clinic

Neuroscience, University of Palermo, Palermo, Italy of a statistically significant difference both as regards complications incidence, during and after surgery $(p>0.9)$ and as regards overall success ratio of prosthesis implants between the two groups $(p>0.7)$. The possibilities of tracheoesophageal recovery of elderly patients do not show dissimilarities in comparison with the results in younger subjects.

Keywords Laryngectomy - Alaryngeal speech · Laryngeal prosthesis $\cdot$ Aged $\cdot$ Outcome

\section{Introduction}

The awareness of the serious disablement related to larynx removal induced the laryngologists, since the beginning of this surgery, to look for different solutions of functional recovery.

From very first attempts carried out by Billroth and Gussenbauer, in 1874, to more recent times, experimental and clinical researches aimed to the possibility of functional voice recovery have followed different paths. Nowadays, these developments have brought post laryngectomy rehabilitation to three main methods: esophageal speech education, electronic aids and tracheoesophageal puncture with prosthesis placement.

Undoubtedly one of the most widely used methods of vocal rehabilitation of laryngectomee is esophageal speech. Even though it does not reach the perfection of the normal voice, in qualitative terms, it is socially effective, allowing understandable communication also at distance. Unfortunately, literature data suggest that only a low percentage can obtain good phonatory results. This is mainly due to anatomical and functional alterations in upper aerodigestive tract, inadequate distribution of logopedic schools over 
the territory, lack of motivation or psychological refusal of esophageal phonation [1].

The most widely used electronic devices are nowadays the so-called laryngophones, improperly addressed as artificial larynxes. Essentially, they are made up of an oscillator device that, put on the neck skin, causes a vibration and this is spread through hypopharyngeal walls that become the source of the sound. The advantages linked to the use of such devices, in comparison with the use of the esophageal voice, are substantially represented by immediate recovery of the voice, without needing logopedic rehabilitation and by voice restoration even in patients with gastroesophageal problems. Moreover, this method is accepted also by patients who refuse esophageal voice because of psychological motivations. Main drawbacks are linked to the production of unnatural voice and to the need for using a hand, such elements largely limit the use of this methodic compared to the more natural esophageal voice.

Tracheoesophageal puncture and placement of modern voice prosthesis has found wide usage throughout the 1980s when Blom and Singer introduced an innovative model of prosthesis. That, when positioned in a tracheoesophageal fistula, which was created on purpose during laryngectomy (primary TEP) or subsequently made (secondary TEP), allowed the production of voice with greater intelligibility and naturalness in comparison with esophageal voice. Therefore, it is clear that, today, laryngectomees or patients who are going to undergo laryngectomy must be properly informed, during presurgical counseling, about the various chances of voice rehabilitation, underling both advantages and limits related to the aforementioned methods of voice recovery.

This is particularly important especially if it is considered that recent data suggest an age range for patients being referred to total laryngectomy (TL) between the 6th and the 7 th decades of life [2-7]. According to this point of view, patients over 70 years of age deserve particular care because they are not only mutilated in their vocal function, but even lack, for age reasons, appropriate motivations to any voice recovery. In addition, they present several comorbidities that could severely limit the choice of a more profitable treatment, such as prosthesis implant.

The aim of our work has been to evaluate the different options of tracheoesophageal voice rehabilitation in over 70-year-old patients who had undergone laryngectomy, assessing advantages and drawbacks of this method of vocal recovery.

\section{Materials and methods}

At the Otorhinolaryngological Clinic of the Department of Medical and Surgical Specialties of the University of
Catania a retrospective study, regarding the period between 2000 and 2010, has been carried out. This work has included 40 subjects, all aged more than 70 years old (range 70-85, mean 77.5), who have undergone laryngectomy and who have subsequently been referred to voice rehabilitation. The group was made of 32 male patients and 8 female patients. As regards disease control: 16 patients underwent total laryngectomy (TL), 11 patients underwent TL and neck dissection (ND), 11 patients underwent TL and neck dissection (ND) and postoperative radiotherapy (PORT), 2 patients underwent TL with partial hypopharyngectomy without reconstruction (Table 1). In particular, it has been realized a phonatory fistula between trachea and esophagus with prosthesis positioning by means of a primary puncture (primary TEP) in 18 cases and it has been realized a secondary puncture (secondary TEP) in 22 cases (Table 2); all the tracheoesophageal punctures were performed after appropriate assessment of motivations, local oncological conditions, comorbidities, and psychic and physical fitness, both local and systemic.

Procedures for primary and secondary TEP were carried out according to those described by other authors [8, 9]. All patients were rehabilitated with indwelling Provox voice prostheses (Atos Medical AB, Hörby, Sweden).

The local institutional review board approved the study protocol and informed consent was obtained from the patients.

In all patients, a surgical refinements was performed at the time of TL for prevention of hypertonicity of the neoglottis, microstoma or deep stoma and pseudo-vallecula formation. In particular was performed a short cricopharyngeal myotomy, a tracheostoma construction by suturing the skin flap as far back as possible to the lateral-posterior tracheal cartilage and sectioning of the sternal head of the sternocleidomastoid muscles, and a pharyngeal reconstruction by closure in T-shape and constrictor muscle closure across the midline and to base of tongue muscles.

In secondary TEP, it was carried out a presurgical evaluation of pharyngoesophageal segment (PES) tonicity by means of insufflation test as described by Blom et al. [10]. A swallowing videofluoroscopy was eventually

Table 1 Treatment options

\begin{tabular}{lcc}
\hline & Study group & Control group \\
\hline TL & 16 & 13 \\
TL and ND & 11 & 11 \\
TL and ND and PORT & 11 & 12 \\
TL and partial hypopharyngectomy & 2 & 3 \\
& 40 & 39
\end{tabular}

$T L$ total laryngectomy, $N D$ neck dissection, PORT postoperative radiotherapy 
Table 2 Puncture type

\begin{tabular}{lll}
\hline & Study group & Control group \\
\hline Primary TEP & 18 & 17 \\
Secondary TEP & 22 & 22 \\
\hline
\end{tabular}

TEP tracheoesophageal puncture

performed to rule out a hypertonicity or spasm of the PES. Patients with hypertonicity or spasm of the PES did not undergo voice prosthesis insertion and were excluded from the examination group.

The results gathered in the selected 40 patients, all over 70 years old, were compared with the data obtained from a group made of 39 patients, under 70 years of age that therefore represented our control group. In the latter group, age range was between 40 and 69 years old, with a mean of 54.5 years, sex distribution was 30 males and 9 females, and treatment options carried out were represented by: 13 cases of TL, 11 cases TL and ND, 12 cases TL and ND and PORT, 3 cases of TL with partial hypopharyngectomy without reconstruction (Table 1). In this group has been realized a phonatory fistula between trachea and esophagus with prosthesis positioning by means of a primary puncture (Primary TEP) in 17 cases and it has been realized a secondary puncture (Secondary TEP) in 22 cases (Table 2).

The results have been obtained analyzing complications and problems during and after the surgery, short-term success, which was given by vocal recovery after 3 months, and long-term success, which was evaluated no sooner than 2 years after surgical procedures, according to the parameters use, quality and care as they are stated by the Harrison-Robillard-Schultz (HRS) TEP rating scale [11] (Table 3).

Categorical data were presented as frequency of occurrence, and the $\chi^{2}$ test and the Fisher's test were used to assess differences between groups.

\section{Results}

In primary TEP, no complications during and after surgery were observed. In four cases, it was seen an instability of tracheostomy diameter that prevented cannula removing and needed for adequate management a fenestrated Larytube use, making particularly difficult the counseling for tracheoesophageal voice learning and reducing vocal intelligibility. In two other cases, both referred to PORT, was observed a reduction of vocal intelligibility with strain voice, unsuitable for functional use during conversation. In this group, short-term success was $67 \%$.

In 22 cases, who underwent secondary TEP, time between laryngectomy and prosthesis implant was on an average 15.2 months, with a range from 14.2 to 16 months. In this group, two cases of tracheoesophageal fistula infection were recorded with peristomal cellulitis, and this made necessity to immediate removal of prosthesis and antibiotic therapy that has led to subsequent healing of tracheoesophageal tissues. Moreover, another case of postsurgical infection of the fistula was observed, due to inappropriate diameter of prosthesis and this was treated with longer prosthesis use. Lastly, intelligibility was poor and unsuitable for conversation in five cases, thence determining a short-term success of $64 \%$.

As far as long-term success is concerned the parameters taken into consideration were use, quality and care as stated by the HRS TEP rating scale [11] (Table 3). Tracheoesophageal rehabilitation was considered successful for total overall score of 11 or higher. After 2 years from TEP, the long-term success (HRS overall score $\geq 11$ ) was $82.5 \%(33 / 40)$, with $78 \%$ in primary TEP and $86 \%$ in secondary TEP (Table 4). The mean of HRS rating scale was 11.4 in patients with primary TEP and 11.5 in patients with secondary TEP.

In the control group, among the 17 patients referred to primary TEP was recorded a microstoma in three cases and the absence of vocal function in other three cases, with a short-term success of $65 \%$. In secondary TEP the time between laryngectomy and prosthesis implant was on average 12.2 months, with a range from 10.1 to 14.3 months and, in this group, were observed two case of mediastinitis and in two cases was seen a peristomal cellulitis, both treated with antibiotic therapy and prosthesis removal. Moreover, two cases of intelligibility reduction were recorded, determining a short-term success rate of $73 \%$.

After 2 years from TEP, the long-term success (HRS overall score $\geq 11)$ was $77 \%$ (30/39), with $70 \%$ in primary TEP and $82 \%$ in secondary TEP (Table 4). The mean of HRS rating scale was 11.7 in patients with primary TEP and 12 in patients with secondary TEP.

The evaluation of the results has shown the absence of a statistically significant difference both as regards complications incidence, during and after surgery $(p>0.9)$ (Table 5) and as regards overall success ratio of prosthesis implants between the two groups $(p>0.7)$ (Table 4).

The success rate was $86 \%$ without PORT use and $73 \%$ with PORT among patients over 70 years old and $78 \%$ without PORT and $75 \%$ with PORT in the control group. The mean of HRS rating scale of patients, who underwent PORT was 11.2 points and the HRS rating scale of patients, who did not undergo PORT was 11.4 points. In addition, comparing PORT exposed subgroups, no statistically significant difference can be found between the two groups $(p>0.8)$ (Table 6). 
Table 3 Harrison-Robillard-Shultz TEP rating scale [11]

A. Use

The degree of use of tracheoesophageal speech

1. Never uses tracheoesophageal speech

2. Uses tracheoesophageal speech less than $50 \%$ of the time

3. Uses tracheoesophageal speech $50 \%$ to $80 \%$ of communicative attempts

4. Uses tracheoesophageal speech manually occluded as main means of communication

5. Uses tracheoesophageal speech with tracheostoma valve as main means of communication

B. Quality

The ease of production and intelligibility of speech as determined by fluency and ability to occlude

1. Unable to get sound; no use of pulmonary air for speech

2. Voice is too strained or too breathy to permit functional use in conversation (may interfere with intelligibility); includes whispered speech

3. Stoma, more often than not, is poorly occluded with resultant air escape that interfere with intelligibility or is a distraction to the listener

4. Voice is mildly stained or mildly breathy, but continuous use in conversation is possible; occlusion is generally good; speech is intelligible

5. Voice is easily produced; occlusion is good; speech is intelligible

C. Care

Patient independence of medical or other health care professionals (includes speech/language pathologist, nurse, community, worker) for four behaviors: (1) remove and insert prosthesis (2) clean and sterilize prosthesis (3) recognize problems and seek help immediately if needed, and (4) order supplies

1. Unable to do any of the four behaviors

2. Independent for any one of four behaviors

3. Independent for any two of four behaviors

4. Independent for any three of four behaviors

5. Independent for all four behaviors

Table 4 Success rate comparison in different age groups

\begin{tabular}{lrr}
\hline & $>70$ & $<70$ \\
\hline HRS $>11$ & 33 & 30 \\
HRS $<11$ & 7 & 9 \\
& 40 & 39 \\
\hline
\end{tabular}

$p>0.7$

HRS Harrison-Robillard-Schultz rating scale

Table 5 Complication incidence

\begin{tabular}{lrr}
\hline & $>70$ & $<70$ \\
\hline Secondary TEP with complications & 3 & 4 \\
Secondary TEP without complications & 19 & 18 \\
& 22 & 22 \\
\hline
\end{tabular}

$p>0.9$

TEP tracheoesophageal puncture

\section{Discussion}

There is no evidence of clinical contraindication to referring geriatric patients to esophageal rehabilitation or to using electric devices. Nevertheless, the latter option should be suggested only after a failure of esophageal voice
Table 6 Success rate comparison related to PORT

\begin{tabular}{lrr}
\hline & $>70$ & $<70$ \\
\hline Without PORT & 25 & 21 \\
PORT & 8 & 9 \\
& 33 & 30 \\
\hline
\end{tabular}

$p>0.8$

PORT postoperative radiotherapy

learning or because of absolute contraindications to tracheoesophageal puncture.

In this context, older patient would seem more easily addressed to esophageal speech education. However, even this is not absolutely free of failure, that is mainly related to fickleness in logopedic training or cricoesophageal muscles and inferior pharyngeal constrictor hypertonicity or psychological refusal of this method of vocal restoration.

On the other side, the choice of esophageal rehabilitation, in these patients, seems to be linked particularly to the need for minimizing the drawbacks of house management of voice prosthesis that, even if positioned by means of primary TEP, requires several clinical efforts of patients. Furthermore, in case of secondary TEP, disadvantages related to a new surgical procedure would gain greater importance, with several complications described such as 
paraesophageal abscesses, esophageal perforations and stenosis, cellulitis, cervical spine fractures, osteomyelitis that luckily are rare [12] (16\% in our study group and $18 \%$ in the control group).

Nonetheless, even in this group of patients, the choice of the rehabilitation method that gives the best vocal result should point towards the voice prosthesis. In addition, considering that possible limits of use are only theoretical if the geriatric patients are found properly suitable as regards psychophysical conditions and motivation, which are the basis needed for obtaining rehabilitation success in tracheoesophageal recovery [13].

Besides, in this context, the decision between primary and secondary TEP could be hard. Indeed if primary TEP eliminates the already rare complications related to the surgical procedure of secondary TEP, on the other hand in the latter we do not have adequate prognostic information about rehabilitation success, linked to possible stenosis or unexpected hypertonicity and/or spasm of pharyngoesophageal segment. These are particularly notable in patients who have undergone radiotherapy, that should be treated first with appropriate logopedic training, before using more complex options such as chemical denervation, botulinum toxin injection, secondary myotomy of pharyngeal constrictor with, if necessary, unilateral neurectomy of pharyngeal plexus. The choice, in such group of patients, of primary implant should be considered according to the aforementioned problems, and the need for a primary myotomy of the cricopharyngeal muscle during laryngectomy, reducing the chances of hypertonicity and/or spasm, which could lead to treatment failure and to further procedures, interfering with the motivation of an older patient [3].

Hence, the choice of secondary TEP, in such patients, could seem more favorable allowing a stabilized and more suitable clinical condition, as far as pharyngoesophageal tonicity, stoma diameter and radiotherapy results are concerned, which could improve the success rate in rehabilitation of laryngectomized patients over the age of 70 .

Data addressing the tracheoesophageal vocal recovery in older patients are given by Chone et al. [3] in a study enlisting 71 patients with a success rate of $96 \%$ for primary TEP and $75 \%$ in secondary TEP, concluding that age does not hinder rehabilitation success.

As regards prosthesis duration, data from literature review show, surprisingly, in case series by Op de Coul et al. [2] about 318 patients from 29 to 88 years of age, a mean device life of 99 days in the group aged under 60 years, of 111 days in the group aged between 60 and 70 years and 147 days in the group aged over 70 years. Furthermore, in this report was shown how different parameters, such as age, sex, type and stage of tumor, myotomy, neurectomy, do not interfere with possible fistula pathology related to prosthesis presence. In contrast, a worse vocal result in the voice quality assessments score (MVQ) was found in subject over 70 years of age in comparison to younger ones. This result was due to both a larger surgical procedure, which needed, in higher percentage, hypopharyngeal sacrifice and a functional situation of lungs that was definitely worse than in younger patients [2].

An important addition is given by Boscolo-Rizzo et al. [14] who reported, in a group of 93 patients, a success rate of $97.5 \%$ among subjects under 61 years of age and a success rate of $69.8 \%$ among subjects over 61 years of age, assuming that younger age improves vocal rehabilitation in primary TEP, but not in secondary TEP.

More figures about prosthesis life are reported by Lequeux et al. [15] about 38 prosthesis patients in whom it was recorded a mean device life of 340 days (Provox) among patients with an age under 60 years in comparison to 392 days among patients, with the same prosthesis, aged over 60 years; moreover, it was observed a mean device life of 217 days (Provox II) among patients with an age under 60 years in comparison to 201 days among patients, with the same prosthesis, aged over 60 years.

Our results in the two groups give a mean device life of 320 days (first generation Provox) and 355 days (second generation Provox) in the study group compared with 270 days (first generation Provox) and 315 days (second generation Provox) in the control group. This is justified by a lower fungal and bacterial aggression in geriatric patients.

Other interesting observation can be obtained by scientific contribution of Moerman et al. [16] about surgical closure of fistula in 12 patients of whom 9 above 70 years of age. In this work, it was shown that the main cause was the lack of use of vocal prosthesis.

Some data underlining the importance of regular use, in more than 70-year-old prosthetic patients, of devices, such as heat/moist exchange to reach the best lung recovery and/ or the use of automatic valve for free-hand phonation are described by Gonzales-Botas et al. [17, 18], who studied 40 patients with an age between 33 and 78 years of age and by Lorenz et al., who enlisted 24 patients with age ranging from 42 to 78 years.

\section{Conclusions}

According to the clinical experience, obtained by studying patients analyzed in this work and in relation with the observations carried out on previously implanted patients that are now more than 70 years, we have found no significant differences, linked to the age, in surgical procedures or in prosthesis life or in fistula pathology that could hinder tracheoesophageal rehabilitation. 
Therefore, on the basis of aforementioned data and in the light of current literature review, it seems clear that the rehabilitation choice should be linked mainly to patients' will, to whom all different options should be thoroughly described, properly addressing both indications and limits. In this context, the possibilities of tracheoesophageal recovery of geriatric patients do not show dissimilarities in comparison with results in younger subjects, on condition that there are adequate motivations and psychophysical suitability, allowing a high rate of short- and long-term success.

Conflict of interest None.

\section{References}

1. Ferrer Ramírez MJ, Guallart Doménech F, Brotons Durbán S, Carrasco Llatas M, Estellés Ferriol E, López Martínez R (2001) Surgical voice restoration after total laryngectomy: long-term results. Eur Arch Otorhinolaryngol 258(9):463-466

2. Op de Coul BM, Hilgers FJ, Balm AJ, Tan IB, van den Hoogen FJ, van Tinteren H (2000) A decade of postlaryngectomy vocal rehabilitation in 318 patients: a single institution's experience with consistent application of provox indwelling voice prostheses. Arch Otolaryngol Head Neck Surg 126(11):1320-1328

3. Chone CT, Gripp FM, Spina AL, Crespo AN (2005) Primary versus secondary tracheoesophageal puncture for speech rehabilitation in total laryngectomy: long-term results with indwelling voice prosthesis. Otolaryngol Head Neck Surg 133(1):89-93

4. Hall FT, O'Brien CJ, Clifford AR, McNeil EB, Bron L, Jackson MA (2003) Clinical outcome following total laryngectomy for cancer. ANZ J Surg 73(5):300-305

5. Iseli TA, Agar NJ, Dunemann C, Lyons BM (2007) Functional outcomes following total laryngopharyngectomy. ANZ J Surg 77(11):954-957

6. Cakli H, Ozudogru E, Cingi E, Kecik C, Gürbüz K (2005) Near total laryngectomy: the problems influencing functions and their solutions. Eur Arch Otorhinolaryngol 262(2):99-102
7. Bernáldez R, García-Pallarés M, Morera E, Lassaletta L, Del Palacio A, Gavilán J (2003) Oncologic and functional results of near-total laryngectomy. Otolaryngol Head Neck Surg 128(5): 700-705

8. Blom ED, Hamaker RC (1996) Tracheoesophageal voice restoration following total laryngectomy. In: Myers EN, Suen JY (eds) Cancer of the head and neck, 3rd edn. Saunders, Philadelphia, pp 839-852

9. Kao WW, Mohr RM, Kimmel CA, Getch C, Silverman C (1994) The outcome and techniques of primary and secondary tracheoesophageal puncture. Arch Otolaryngol Head Neck Surg 120(3): 301-307

10. Blom ED, Singer MI, Hamaker RC (1985) An improved esophageal insufflation test. Arch Otolaryngol 111:211-212

11. Shultz JR, Harrison J (1992) Defining and predicting tracheoesophageal puncture success. Arch Otolaryngol Head Neck Surg 118:811-816

12. Garth RJ, McRae A, Rhŷs Evans PH (1991) Tracheo-oesophageal puncture: a review of problems and complications. J Laryngol Otol 105(9):750-754

13. Pawar PV, Sayed SI, Kazi R, Jagade MV (2008) Current status and future prospects in prosthetic voice rehabilitation following laryngectomy. J Cancer Res Ther 4(4):186-191

14. Boscolo-Rizzo P, Zanetti F, Carpené S, Da Mosto MC (2008) Long-term results with tracheoesophageal voice prosthesis: primary versus secondary TEP. Eur Arch Otorhinolaryngol 265: $73-77$

15. Lequeux T, Badreldin A, Saussez S, Thill MP, Oujjan L, Chantrain G (2003) A comparison of survival lifetime of the Provox and the Provox 2 voice prosthesis. J Laryngol Otol 117(11): 875-878

16. Moerman M, Vermeersch H, Heylbroeck P (2004) A simple surgical technique for tracheoesophageal fistula closure. Eur Arch Otorhinolaryngol 261(7):381-385

17. Lorenz KJ, Groll K, Ackerstaff AH, Hilgers FJ, Maier H (2007) Hands-free speech after surgical voice rehabilitation with a Provox voice prosthesis: experience with the Provox FreeHands HME tracheostoma valve system. Eur Arch Otorhinolaryngol 264(2):151-157

18. Herranz González-Botas J, Suárez T, García Carreira B, Martínez Morán A (2001) Experience with the HME-Provox Stomafilter in laryngectomized patients. Acta Otorrinolaringol Esp 52(3): 221-225 